TITLE:

\title{
A halation reduction method for high quality images of tomato fruits in greenhouse
}

\section{$\operatorname{AUTHOR}(\mathrm{S}):$}

Cheng, Xiao; Kondo, Naoshi; Ogawa, Yuichi; Shiigi, Tomoo; Widodo, Slamet; Watanabe, Yuichi; Fujiura, Tateshi

\section{CITATION:}

Cheng, Xiao ... [et al]. A halation reduction method for high quality images of tomato fruits in greenhouse. Engineering in Agriculture, Environment and Food 2015, 8(4): 200-206

\section{ISSUE DATE:}

2015-10

URL:

http://hdl.handle.net/2433/210027

\section{RIGHT:}

(c) 2015. This manuscript version is made available under the CC-BY-NC-ND 4.0 license

http://creativecommons.org/licenses/by-nc-nd/4.0/; The full-text file will be made open to the public on 1 October 2017 in accordance with publisher's 'Terms and Conditions for Self-Archiving'.; This is not the published version. Please cite only the published version.; この論文は出版社版でありません。引用の際には出版社版をご確認ご利用ください。 


\title{
A Halation Reduction Method for High Quality Images of Tomato
}

\author{
Fruits in Greenhouse*
}

\author{
Xiao CHENG $^{* 1}$, Naoshi KONDO ${ }^{* 2}$, Yuichi OGAWA ${ }^{* 1}$, Tomoo SHIIGI ${ }^{* 1}$, Slamet WIDODO ${ }^{* 1}$, \\ Yuichi WATANABE ${ }^{* 1}$, Tateshi FUJIURA ${ }^{* 1}$
}

\begin{abstract}
Halation is one of the serious problems for machine vision system which could cause color information lost of image. Images for tomato fruits in greenhouse with different rotational angle of polarizing filter (PL filter) were acquired and combined into one result image to eliminate multiple halation areas from sunlight and reflected light. An image acquisition system composed of a CCD camera and a stepping motor was used to acquire images. The multiple halation areas could be eliminated in the condition with a certain angle of incidence and color representation in result image was improved. The reasonable result shows the feasibility to eliminate multiple halation areas by this method.
\end{abstract}

[Keywords] halation, machine vision, PL filter, HSI color space, image processing, Brewster’s angle, tomato

\section{Introduction}

A machine vision system is one of the important sensing devices for precision agriculture (Kondo et al., 2006). Using machine vision as monitoring system farmers can obtain information about plant conditions and health which helps them make decisions for plant cultivation (LEE, 2008). Color information is commonly used to analyze the plant health conditions and the quality. The machine vision technique in the field applications is to overcome the often unpredictable and non-uniform (shadows in the field of view) outdoor illumination because unpredictable and nonuniform illumination in the field of view directly affect the captured image quality (Hong Y et al., 2011). In this paper, halation is defined as the high intensity areas in the image caused by specular reflected light on the surface of plants (Nishiwaki et al., 2006). To acquire an optimal quality image, halation is one problem that can cause loss of color information. Due to this problem, it is not easy to get correct color information for defect detection and quality classification under natural light condition.

Since halation is a kind of polarized light coming from the specular reflection of sunlight. Polarizing filters (PL filter) are effective to reduce halation when they are set both in front of the light source and of the camera and the PL
25 filters forms an angle of $90^{\circ}$. However, this method is not 26 effective in an outdoor environment because the light source 27 position changes over time and varies from place to place. 28 Nishiwaki et al., (2006). tried to reduce halation on leaves 29 of a coffee plant by using two PL filters and one frame box 30 covered by a polarizing film in the field. The first PL filter 31 is a polarizing film covering a frame box in the field. This 32 first PL filter allows only horizontally oriented polarized 33 sunlight to reach the plant surface. The second PL filter is 34 set in front of a camera and only the vertical component of 35 light passes. The camera was installed inside the box. The 36 images were analyzed by an RGB model and a HSV model 37 and the halation areas were extracted using a Saturation 38 threshold of 128 . The halation elimination and the color 39 representation results showed that this method is effective 40 to get images without loss of color information. Although 41 their method is promising, two PL filters and the frame box 42 make it not efficient for a real monitoring system. Watanabe 43 et al., (2010) proposed a method to minimize the halation 44 area in the field by rotating the PL filter to a specific angle 45 calculated on the basis of solar altitude, solar azimuth and 46 the camera orientation. It is still difficult to use this method 47 in a greenhouse due to multiple halation areas problem. 48 Reflected light from plastic walls or from metallic

* $\quad$ Partly presented at $127^{\text {th }}$ JSAM Kansai branch meeting in March 2012

*1 JSAM Member, Graduate School of Agriculture, Kyoto University, Kitashirakawa-Oiwakecho, Sakyo-ku, Kyoto 606-8502, Japan

*2 JSAM Member, Corresponding author, Graduate School of Agriculture, Kyoto University, Kitashirakawa-Oiwakecho, Sakyoku, Kyoto 606-8502, Japan. kondonao@kais.kyoto-u.ac.jp 


\begin{tabular}{|c|c|c|c|}
\hline & $\begin{array}{c}\text { Number } \\
\text { of fruit }\end{array}$ & Color & Diameter \\
\hline Material 1 & 1 & Red & $70 \mathrm{~mm}$ \\
\hline Cluster 1 & 3 & Red & $62 \sim 70 \mathrm{~mm}$ \\
\hline Cluster 2 & 4 & 2 Red, 2 Green & $60 \sim 75 \mathrm{~mm}$ \\
\hline Cluster 3 & 6 & Red & $43 \sim 63 \mathrm{~mm}$ \\
\hline
\end{tabular}

18

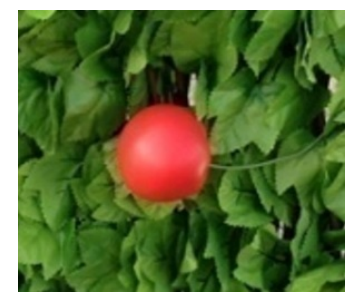

(a) Material 1 for simulation

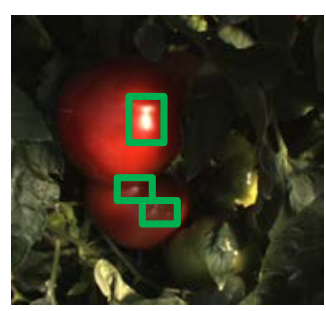

(c) Tomato cluster 2

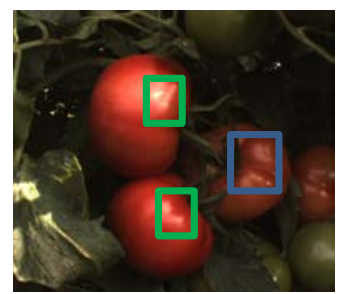

(b) Tomato cluster 1

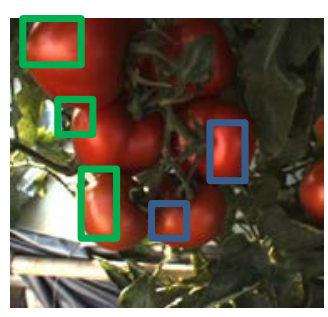

(d) Tomato cluster 3
Fig. 1 Images of the experimental fruits for the simulation test. The distance between camera and tomato was $300 \mathrm{~mm}$. A wall constructed from plastic leaves was used as the background. The images were acquired on May $28^{\text {th }}$, 2012, from 15:00 16:00. The solar altitude was then from $47.57^{\circ}$ to $35.30^{\circ}$. The solar azimuth was from $-84.14^{\circ}$ to $-93.05^{\circ}$ (south is $0^{\circ}$ and southwest is negative degree). In the simulation, sunlight came from the southwest. One mirror was set on east side of the tomato such that the sunlight itself and the reflected light from the mirror caused two halation areas in the images.

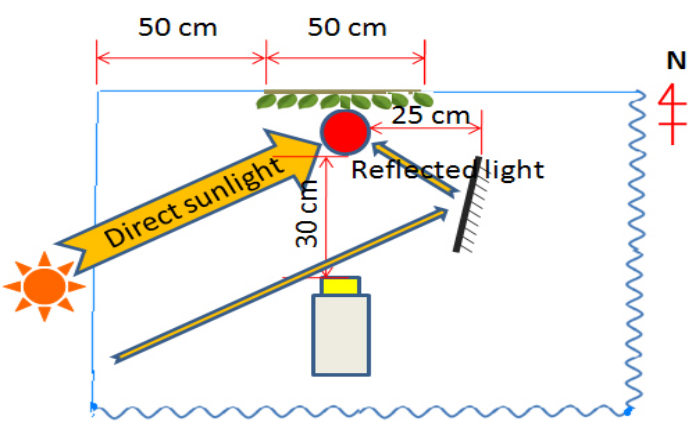

(a) Top view of simulation setup

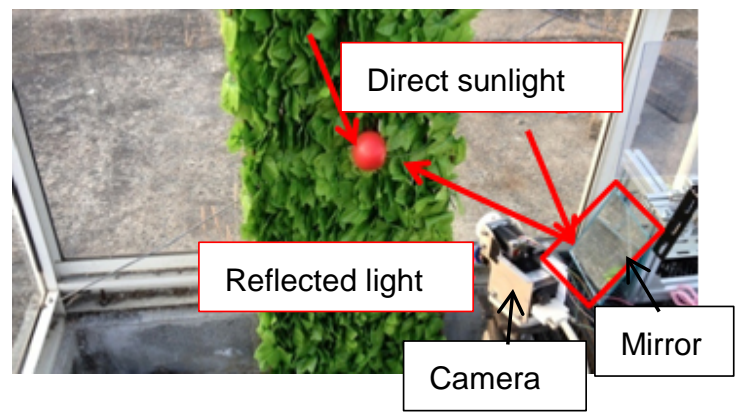

(b) Simulation experiment

Fig. 2 Simulation experiment setup

\section{Greenhouse experiment}

The greenhouse experiment was conducted at the National Institute of Vegetable and Tea Science, Aichi Prefecture. The setup of greenhouse experiment for the tomato clusters is shown in Fig. 3. Images were acquired on Dec $8^{\text {th }}$, 2009. The image acquisition time was (1) 12:00 - 12:12, (2) 14:12 - 14:38 and (3) 15:05 - 15:22 for cluster 1,2 and 3 . The solar altitude was (1) from $32.18^{\circ}$ to $31.95^{\circ}$, (2) from $22.67^{\circ}$ to $19.31^{\circ}$ and (3) from $15.44^{\circ}$ to $12.83^{\circ}$. The solar azimuth was (1) from $-3.87^{\circ}$ to $7.12^{\circ}$, (2) from $-36.52^{\circ}$ to $-41.83^{\circ}$ and (3) from $-46.94^{\circ}$ to $-49.96^{\circ}$. The distance between camera and tomato clusters 1, 2 and 3 were $226 \mathrm{~mm}, 200 \mathrm{~mm}$ and $335 \mathrm{~mm}$, respectively. The arrows in Fig. 3 indicate the optical path for three tomato clusters. Due to sunlight and the light reflected from the plastic wall of the greenhouse, 3 (Fig. 1(b)), 3 (Fig. 1(c)), and 5 (Fig. 1(d)) halation areas could 
CHENG, KONDO, OGAWA, SHIIGI, WIDODO, WATANABE, FUJIURA: A Halation Reduction Method for ${ }^{3}$ High Quality Images of Tomato Fruits in Greenhouse

1 be observed on surface of respectively the tomato cluster

2 1, 2, and 3, as shown in Fig. 1. The areas marked by green

3 and blue box in Fig.1(b), Fig.1(c), Fig.1(d) correspond to

4 halation caused by direct sunlight and reflected light,

5 respectively.

6

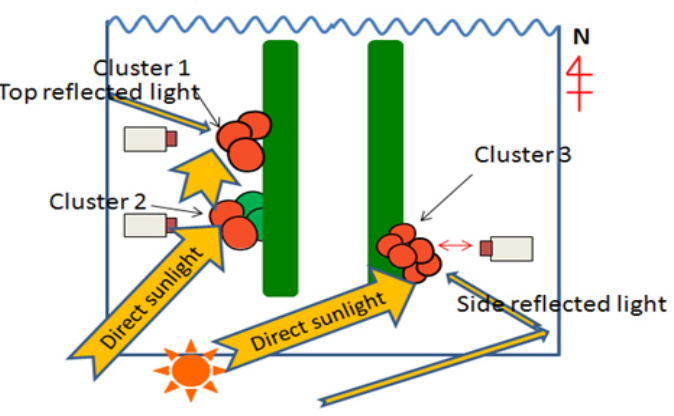

Fig. 3 Greenhouse experiment setup

\section{Measurement equipment and Methods}

\section{Image acquisition system}

Figure 4 shows the image capturing system for the experiments. A CCD camera (VCC-8350CLTS, CIS CO.,Ltd, RGB signal, resolution: 512(H) $\times 480(\mathrm{~V})$, frame rate: $60 \mathrm{fps}$ ) with $6 \mathrm{~mm}$ lens and an image capture board (MTPCI-TL, Micro-Technica) installed in a PC was used to capture tomato images. The camera parameters (shutter speed, gains, white balance) were adjusted by software for the different illumination intensities and color temperatures. A PL filter (with a blue gear diameter: $40 \mathrm{~mm}$, number of teeth: 51, pitch: $2 \mathrm{~mm}$ ) was set in front of camera lens. A stepping motor (CFK525BP2, ORIENTAL MOTOR CO., Ltd, rotational speed: $60 \mathrm{rpm}$ ) (with a white gear diameter: $40 \mathrm{~mm}$, number of teeth: 51, pitch: $2 \mathrm{~mm}$ ) was mounted on the camera. Pulses generated by PC drive stepping motor with subdivided driving to rotate the PL filter with one degree steps. Tomato images can be acquired with different rotational angles of PL filter. After the adjustment, the parameters of camera were set as: F number is 1.2; shutter speed is $1 / 2000 \mathrm{~s}$; gain is $150 \sim 180$.

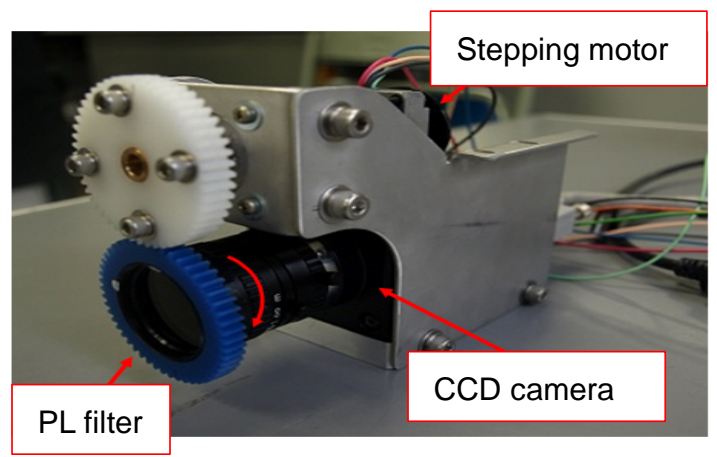

Fig. 4 Image acquisition system
51
The halation is regarded as light synthesized of $P$ polarized light (the component of the electric field parallel to plane of incidence) and $S$-polarized light (the component perpendicular to plane of incidence). Figure 5(a) shows schematic diagram for angle of incidence and Figure 5(b) shows the relation between angle of incidence and reflectance ratio of polarized light. The angle when the P-polarized light reflectance ratio becomes 0 is called Brewster's angle (around $55^{\circ}$ ). Since $S$-polarized light increases monotonically and shows higher ratio than $P$ polarized light anytime, reduction of $S$-polarized light makes less halation. Thus if PL filter can block the $S$ polarized light when angle of incidence is Brewster's angle, halation can be eliminated. Based on this principle, Watanabe et al., (2010) proposed a method to calculate the rotation angle by solar azimuth: $\theta$; solar altitude: $\varphi$; camera orientation: $\psi$. the rotational angle $\gamma$ of PL filter which can minimize halation can be calculated by:

$$
\gamma=\tan ^{-1}\left(\frac{\sin \theta \cos \varphi}{\cos \theta \cos \varphi \sin \psi+\sin \varphi \cos \psi}\right)
$$

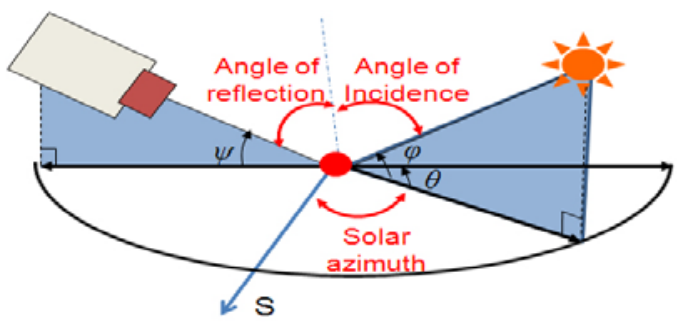

(a) Schematic diagram for angle of incidence

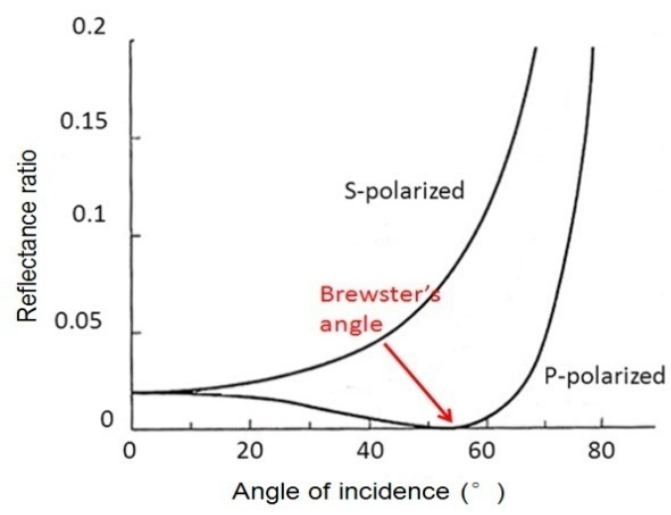

(b) Relation between angle of incidence and reflectance ratio

Fig. 5 Halation minimizing method by using Brewster's angle

Since the multiple halation areas in one image cannot be minimized simultaneously with one rotational angle of the 
1 PL filter, images with different rotational angles were acquired. Previous research (Aibara, 2006) found that halation started to appear on tomato surface after rotating PL filter $7^{\circ}$, which means the rotational step must be less 5 than $7^{\circ}$. In this study, images were acquired with $5^{\circ}$ 6 rotational step intervals from $0^{\circ}$ to $180^{\circ}$ (for a total of 36 7 images acquired per cluster) both in the simulation and greenhouse experiments.

$H= \begin{cases}\theta & \text { if } B \leq G \\ 360-\theta & \text { if } B>G\end{cases}$

$38 \theta=\cos ^{-1}\left\{\frac{\frac{1}{2}[(R-G)+(R-B)]}{\left[(R-G)^{2}+(R-B)(G-B)\right]^{\frac{1}{2}}}\right\}$

$39 s=1-\frac{3}{(R+G+B)}[\min (R, G, B)]$

$40 i=\frac{1}{3 \times 255}[R+G+B]$

$41 S=s \times 100 ; I=i \times 100$

42 The $R, G$ and $B$ are normalized value in the range of [0,

43 1]. To make "Saturation" and "Intensity" easy to interpret,

44 they are multiplied by 100 . So the $H$ (Hue), $S$ (Saturation)

45 and $I$ (Intensity) are in the range of $[0,360],[0,100]$ and $46[0,100]$, respectively.

47

\section{Halation extraction method}

To evaluate the performance of this method, the halation area on the tomato surface in each image must be extracted from the background. In previous research, Nishiwaki et al., (2006) used $S$ value (Saturation) of HSV model to define halation. He defined the halation area in a coffee plant image by a Saturation threshold of 128 (saturation range in his experiment was $[0,255])$. In the present study, a halation extraction method by using normalized image was proposed: (1) The original image was normalized by:

$r=R / \sqrt{R^{2}+G^{2}+B^{2}} \times 255$

$g=G / \sqrt{R^{2}+G^{2}+B^{2}} \times 255$

$b=B / \sqrt{R^{2}+G^{2}+B^{2}} \times 255$

where $r, g$ and $b$ represents normalized value of red, green and blue components, respectively. (2) The halation areas were extracted with threshold: $r>140$ AND $105<g<170$ AND $80<b<170$ AND $R>80$. (3) The halation areas on tomato surface in original image were marked by blue color.

\section{Results and Discussion}

\section{Captured images with different rotational angle}

Figure 7 shows images of four sample types captured using different rotation angles. Each row of images is for one cluster, the columns are for different rotational angle. The number after rotational angle indicates the halation pixels number extracted in each image. The best rotational angle was calculated by the method described in (Watanabe et al., 2010). The first image of four images of each material shows the most halation minimized result by direct sunlight and the PL filter rotational angle. This rotational angle was close to the calculated best rotational angle. The second image of four images of each material shows the most halation maximized result by direct sunlight when PL filter rotational angle was around $90^{\circ}$. The third and fourth images of four images of each material show the most halation minimized and maximized result by reflected light from the structure. Theoretically, the angle change between minimum halation area and maximum halation area should be $90^{\circ}$. However, sun's position changed during image acquisition procedure, so that an error of about $10^{\circ}$ 
occurred. However, even when the PL filter was rotated to near the theoretically best angle, halation still cannot be completely reduced. Multiple halation areas caused by sunlight and reflected light appears alternately. The worst situation appeared for cluster 2, where halation areas were not reduced effectively even for the PL filter rotated to the best angle.
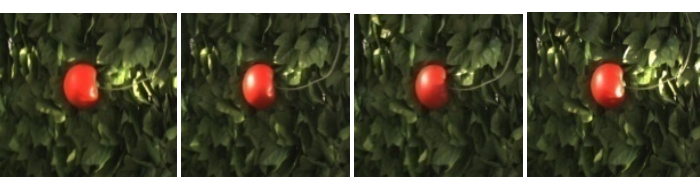

$75^{\circ}(269)$

$175^{\circ}(1574) \quad 15^{\circ}(1203)$

$95^{\circ}(1423)$

(a) Material 1 (Best angle: $69.78^{\circ}$ )
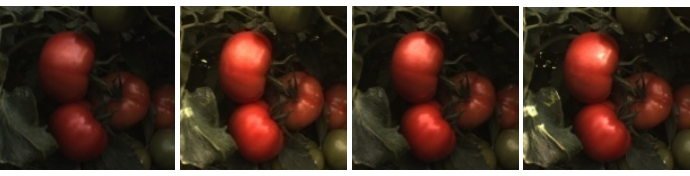

$130^{\circ}(892) \quad 35^{\circ}(3326) \quad 0^{\circ}(2428) \quad 95^{\circ}(3018)$

(b) Cluster 1 (Best angle: $140.59^{\circ}$ )
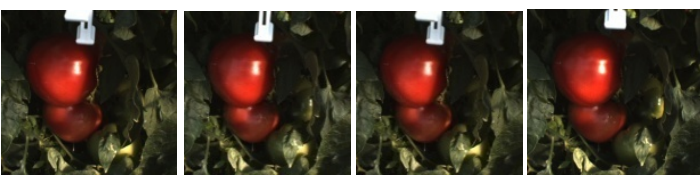

$165^{\circ}(959)$

$75^{\circ}(1128) \quad 120^{\circ}(1014) \quad 30^{\circ}(1123)$

(c) Cluster 2 (Best angle: $159.38^{\circ}$ )
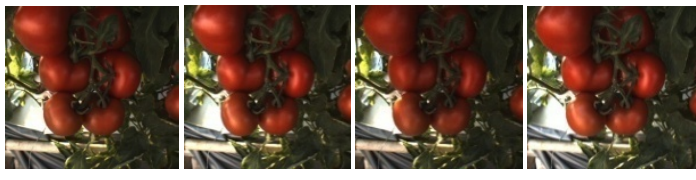

$140^{\circ}(856) \quad 70^{\circ}(1977) \quad 1600^{\circ}(1317) \quad 0^{\circ}(2238)$

(d) Cluster 3 (Best angle: $135.97^{\circ}$ )

Fig. 7 Captured images with four different PL filter rotational angles, theoretically the best rotational angle, and the halation area size.

\section{Halation elimination result}

Figure 8 shows the halation pixel counting result in captured images and in combined result images. The first image of four images of each material shows the result in simulation experiment where the first two images in Fig.8 are the images containing most halation pixels for each cluster. The worst image in simulation counted 1624 pixels as the halation area on the tomato surface (in total the fruit region had 10064 pixels). Compared with the captured image, no halation pixel can be detected in combined image, indicating that both halation areas are successfully eliminated.

Figure 8(b), (c) and (d) show the results in the greenhouse
37 experiments. The halation pixels number in combined 38 image of cluster 1 was significantly reduced. Compared

with the worst quality image which contains 3326 halation pixels, result image only contains 734 halation pixels. The remaining halation pixels were mainly caused by reflected light from behind the camera. All the halation pixels caused by direct sunlight and reflected light from the top wall were completely eliminated. However, one failed example of halation elimination was observed from tomato cluster 2. The halation areas were not reduced effectively in combined image especially for the strong halation spot. This halation spot was caused by direct sunlight from behind the camera and it cannot be reduced even with the PL filter changing to a different angle. In the combined image of cluster 3 , several halation areas were completely eliminated. This confirms that the proposed method is reasonably feasible for halation elimination in practical applications.

The result of tomato cluster 1 and 2 shows that halation caused by light from behind the camera could not be eliminated by the proposed method. The reason for this problem is that halation is composed of $P$-polarized light and $S$-polarized light. The PL filter was only used to eliminate $S$-polarized light. $P$-polarized is eliminated when angle of incidence is around Brewster's angle. When the light comes from behind the camera, the angle of incidence for the tomato is much lower than the Brewster's angle.

For example, when acquiring images of cluster 2, the original setup is shown as left image in Fig. 9 (solar azimuth was $33.6^{\circ}$ and camera faced to east) the angle of incidence was less than $30^{\circ}$ so the halation spot could not be reduced in all images. One solution for this problem is changing the orientation of camera as in the right part of Fig. 9, where the angle of incidence was around $55^{\circ}$. A possible optimum position is proposed from the calculation: if the angle between the light source incident vector and the camera viewing vector is around $70^{\circ}$, then the angle of incidence is for the tomato is around $55^{\circ}$ regardless of the changing solar altitude. When using this halation reduction method, the camera position should be optimized to make the angle of incidence near Brewster's angle. According to experimental results here, halation can be eliminated when the angle of incidence is in the range: $\left[47^{\circ}, 57^{\circ}\right]$. For practical use, because the sun moves from east to west everyday, the camera could be set to face east in the morning and to face west in the afternoon to avoid halation generated by light from behind the camera. 


\section{(1)}

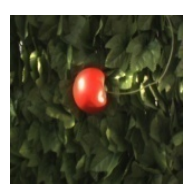

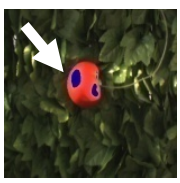

Before (1,624 pixels)

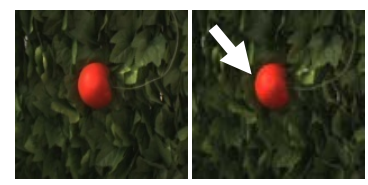

After (0 pixels)

(a) Result of Material 1 (angle $130^{\circ}$ )

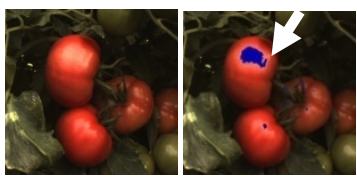

Before (3,326 pixels)

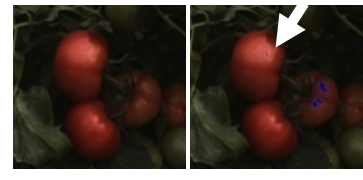

After (734 pixels)

(b) Result of Claster 1 (angle $135^{\circ}$ )
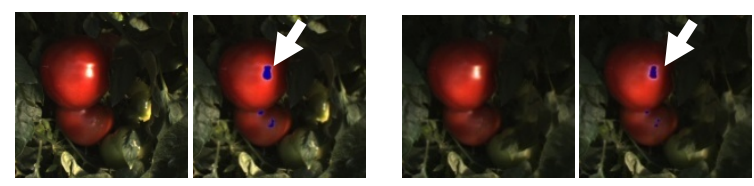

Before (1,209 pixels)

After (861 pixels)

(c) Result of Claster 2 (angle $0^{\circ}$ )

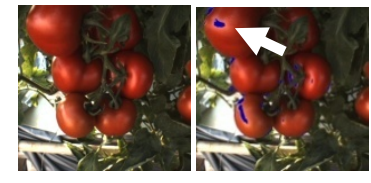

Before (2,960 pixels)

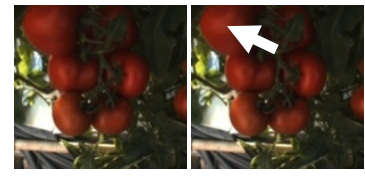

After (0 pixels) (d) Result of Claster 3 (angle $30^{\circ}$ )

Fig. 8 Halation counting result in worst captured image and result image

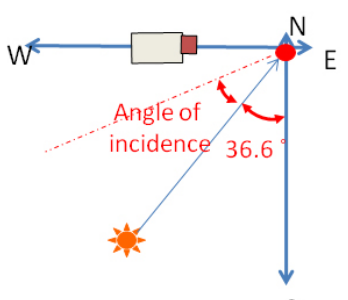

$\mathrm{S}$

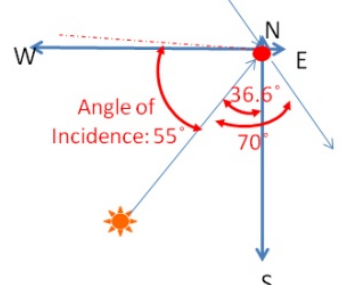

(Original setup)

(Proposed setup)

Fig. 9 Original and proposed setup for acquisition of tomato cluster 2

\section{Color representation}

Figure 10 shows the hue-saturation distribution found on the single tomato (material 1) in the image taken with the PL filter angle at $130^{\circ}$. At this PL filter angle of $130^{\circ}$, the image contains the most halation pixels number among all the captured images and in combined result image. The Figure 10 also gives the hue-saturation distribution of the combined result image. The captured image had many pixels with saturation value less than 40 when compared with the combined result image. This indicates that the color was not properly represented on these halation pixels. It is seen that all saturation values of tomato pixels in the
32 combined result image are above 40. In addition, the 33 standard derivation (SD) of hue and saturation was smaller 34 in the combined result image. Hence, this method removes 35 halation without losing information of the tomato color.

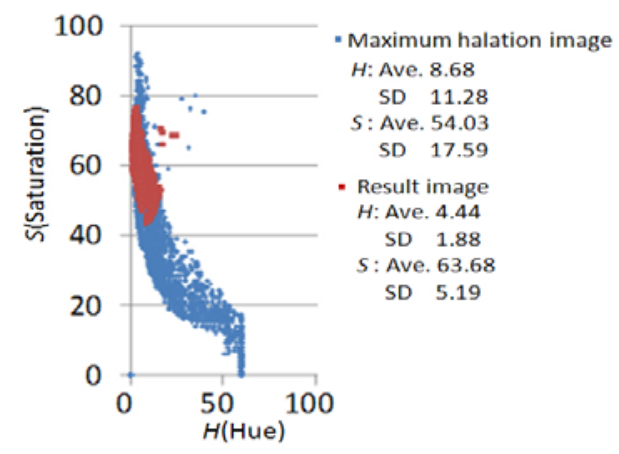

Fig. 10 The hue-saturation distribution of tomato pixels in the laboratory experiment

\section{Conclusion}

A reduction method for multiple halation areas occurring on images taken in a greenhouse was developed by picking the darkest pixels in images taken with different PL filter rotational angles. Multiple halation areas could be eliminated under the condition of a certain angle of incidence. Distribution of hue and saturation values in a combined result image indicated that color representation in this combined result image was much improved. In future studies, usability of Brewster's angle to minimize multiple halation areas in image acquisition in greenhouse needs to be investigated. Two possible solutions are: camera orientation change (location variation) or image acquisition schedule optimization.

\section{References}

Aibara T. 2006. Control of polarizing filter for acquiring high quality images. Graduation thesis. Ehime University, Department of Mechanical Engineering

Gonzalez, R. C. W., E. Richard. 1992. Digital image processing. Reading, Mass: Addison-Wesley.

Hong Y. Jeon, Lei F. Tian and Heping Zhu. 2011. Robust Crop and Weed Segmentation under Uncontrolled Outdoor Illumination. Sensors 2011, Vol.11, pp. 6270-6283

Kondo. N, Namba. K, Nishiwaki. K, Peter P. Ling, M. Monta. 2006. An illumination system for machine vision inspection of agricultural products. 2006 ASABE Annual Meeting

Lee Jong Whan. 2008. Machine vision monitoring system of lettuce growth in a state-of-the-art greenhouse. Modern Physics Letters B, Vol.22, Issue11, pp.953-958

Nishiwaki. K, Kondo. N et al. 2006. Feasibility of using Polarizing Filters to reduce Halation Effects during Image Acquisition in the Field. 2006 ASABE Annual Meeting. 
CHENG, KONDO, OGAWA, SHIIGI, WIDODO, WATANABE, FUJIURA: A Halation Reduction Method for ${ }^{7}$ High Quality Images of Tomato Fruits in Greenhouse

1 Watanabe Y. et al. 2010. Machine vision system for detecting

2 tomato fruits. 2010 ASABE Annual Meeting. 1009874

(Received : X. September. 2012) 